Network Working Group

Request for Comments: 2425

Category: Standards Track
T. Howes

M. Smith

Netscape Communications Corp.

F. Dawson

Lotus Development Corporation

September 1998

\title{
A MIME Content-Type for Directory Information
}

Status of this Memo

This document specifies an Internet standards track protocol for the Internet community, and requests discussion and suggestions for improvements. Please refer to the current edition of the "Internet Official Protocol Standards" (STD 1) for the standardization state and status of this protocol. Distribution of this memo is unlimited.

Copyright Notice

Copyright (C) The Internet Society (1998). All Rights Reserved.

1. Abstract

This document defines a MIME Content-Type for holding directory information. The definition is independent of any particular directory service or protocol. The text/directory content-Type is defined for holding a variety of directory information, for example, name, or email address, or logo. The text/directory content-Type can also be used as the root body part in a multipart/related contentType for handling more complicated situations, especially those in which non-textual information that already has a natural MIME representation, for example, a photograph or sound, is to be represented.

The text/directory Content-Type defines a general framework and format for holding directory information in a simple "type:value" form. We refer to "type" in this context meaning a property or attribute with which the value is associated. Mechanisms are defined to specify alternate languages, encodings and other meta-information. This document also defines the procedure by which particular formats, called profiles, for carrying application-specific information within a text/directory Content-Type can be defined and registered, and the conventions such formats must follow. It is expected that other documents will be produced that define such formats for various applications (e.g., white pages). 
The key words "MUST", "MUST NOT", "REQUIRED", "SHALL", "SHALL NOT", "SHOULD", "SHOULD NOT", "RECOMMENDED", "MAY" and "OPTIONAL" in this document are to be interpreted as described in [RFC-2119].

\section{Table of Contents}

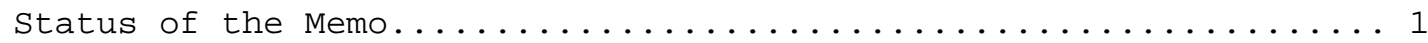

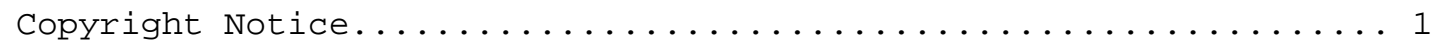

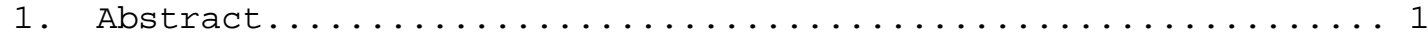

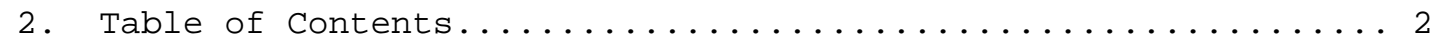

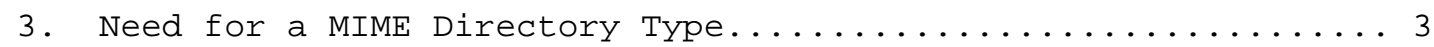

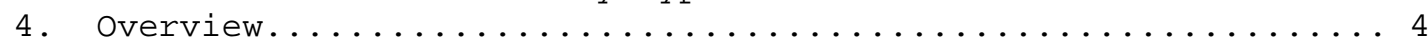

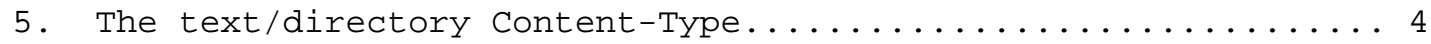

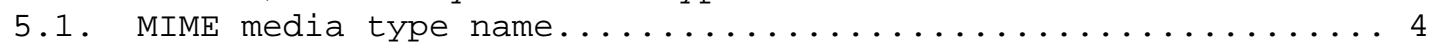

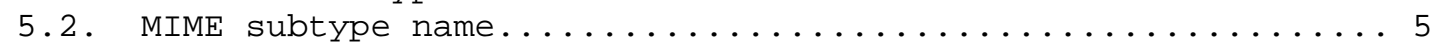

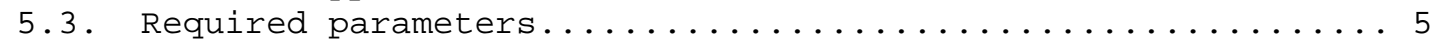

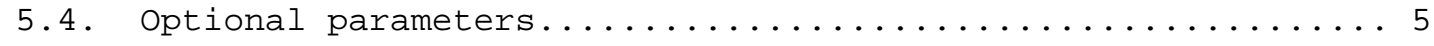

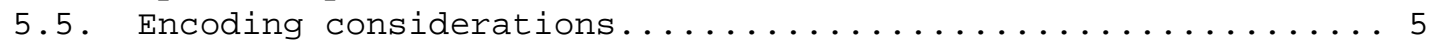

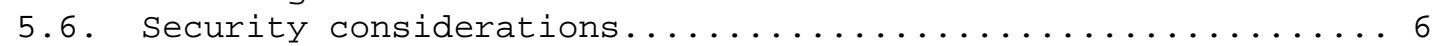

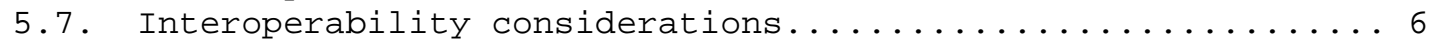

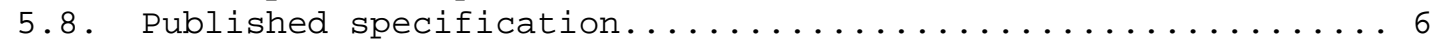

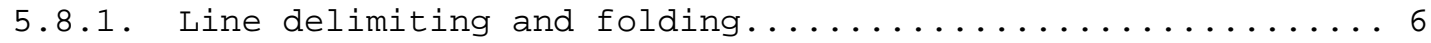

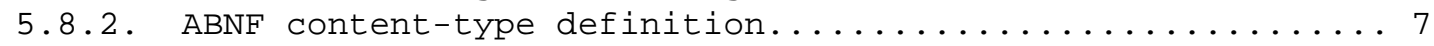

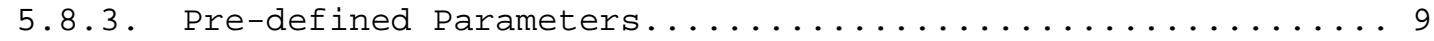

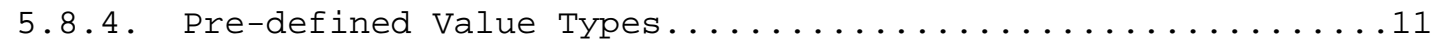

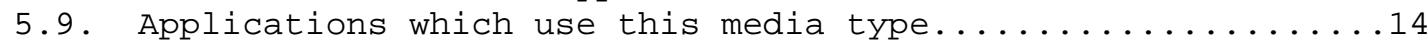

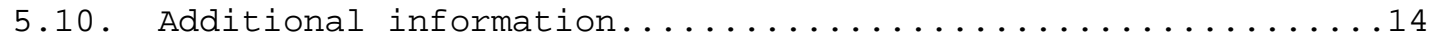

5.11. Person \& email address to contact for further information..14

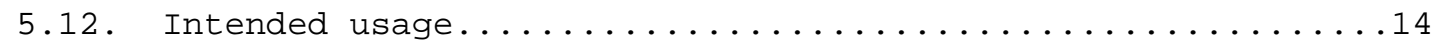

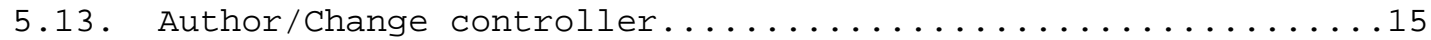

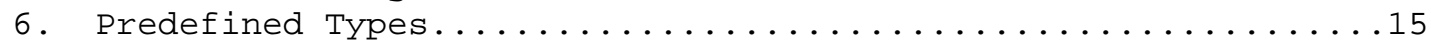

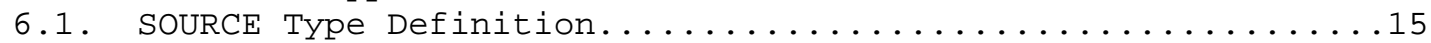

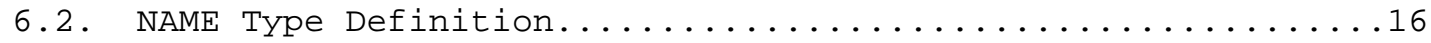

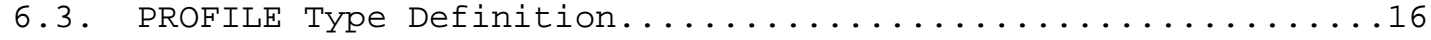

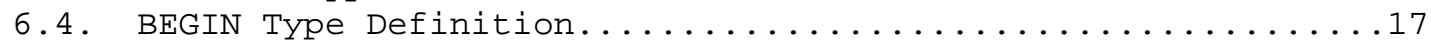

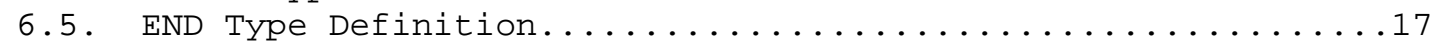

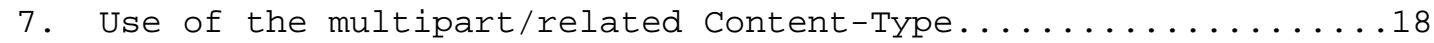

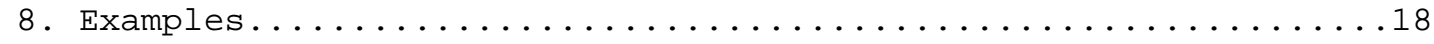

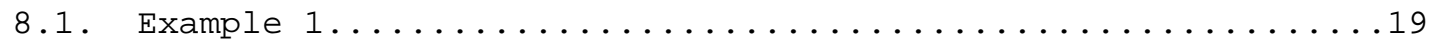

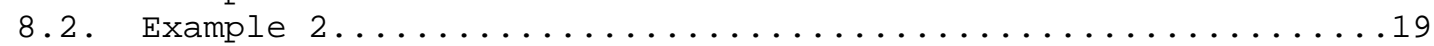

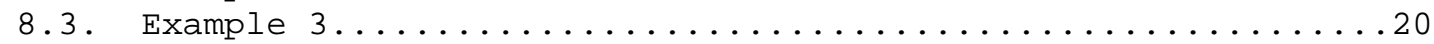

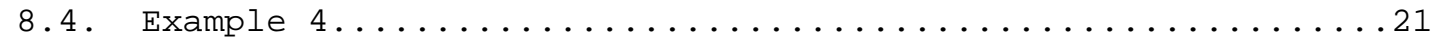

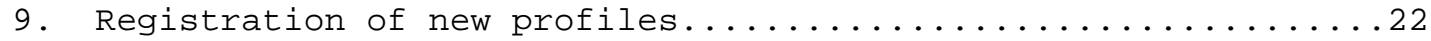

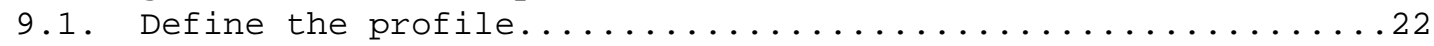

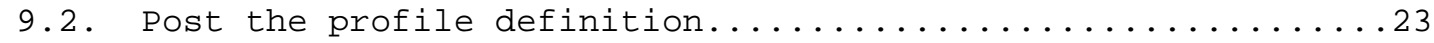

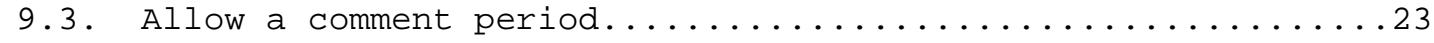

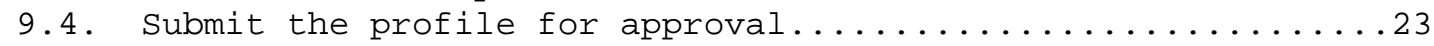

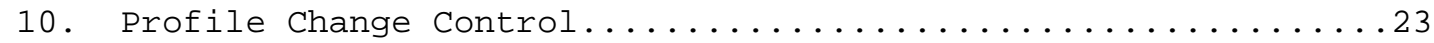




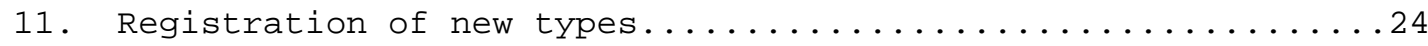

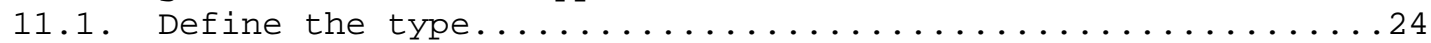

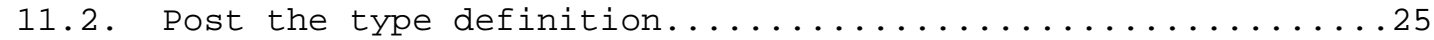

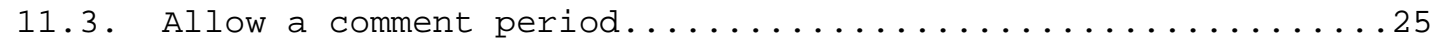

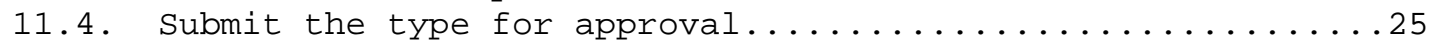

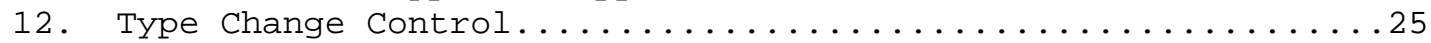

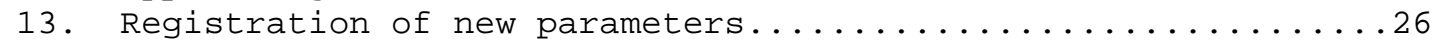

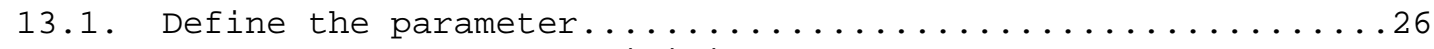

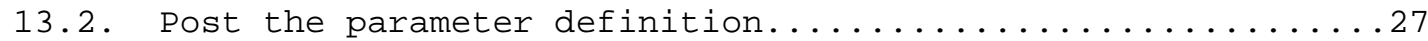

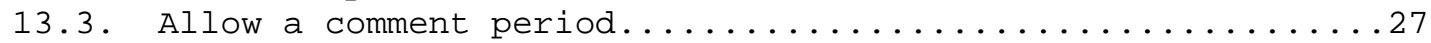

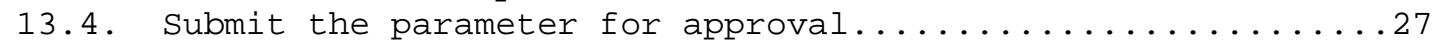

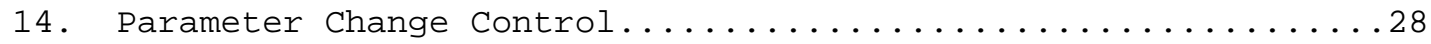

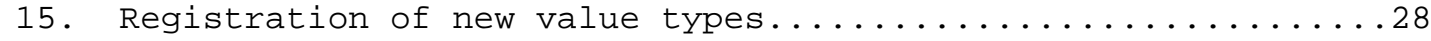

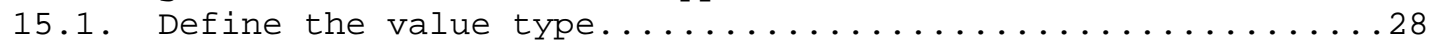

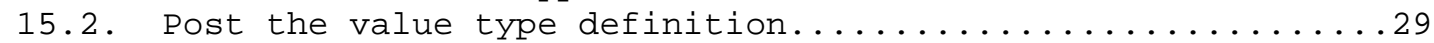

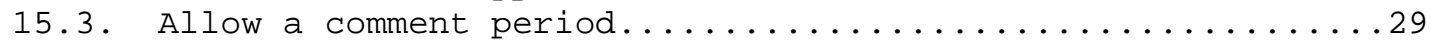

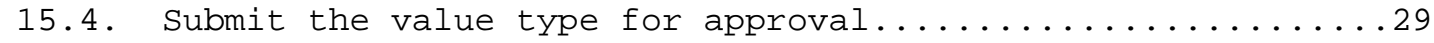

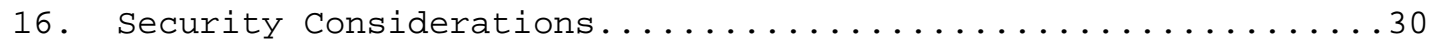

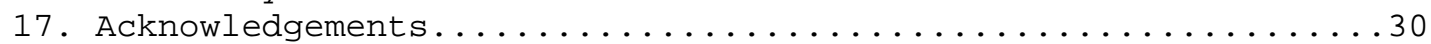

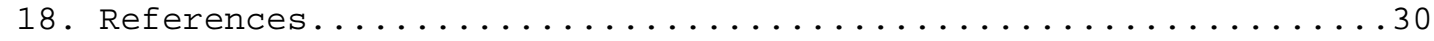

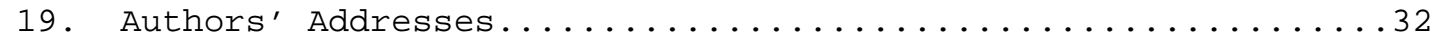

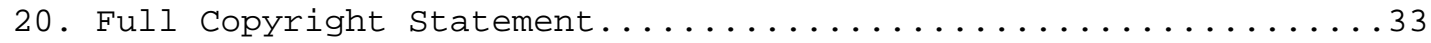

\section{Need for a MIME Directory Type}

For purposes of this document, a directory is a special-purpose database that contains typed information. A directory usually supports both read and search of the information it contains, and can support creation and modification of the information as well. Directory information is usually accessed far more often than it is updated. Directories can be local or global in scope. They can be distributed or centralized. The information they contain can be replicated, with weak or strong consistency requirements.

There are several situations in which users of Internet mail might wish to exchange directory information: the email analogy of a "business card" exchange; the conveyance of directory information to a user having only email access to the Internet; the provision of machine-parseable address information when purchasing goods or services over the Internet; etc. As MIME [RFC-2045, RFC-2046] is used increasingly by other protocols, most notably HTTP, it can also be useful for these protocols to carry directory information in MIME format. Such a format, for example, could be used to represent URC (uniform resource characteristics) information about resources on the World Wide Web, or to provide a rudimentary directory service over HTTP. 


\section{Overview}

The scheme defined here for representing directory information in a MIME Content-Type has two parts. First, the text/directory ContentType is defined for use in holding directory information within a single body part, for example name, title, or email address. In its simplest form, the format uses a "type:value" approach, which should be easily parseable by existing MIME implementations and understandable by users. More complicated situations can be represented also. This document defines the general form the information in the Content-Type should have, and the procedure by which specific types and values (properties) for particular applications can be defined. The framework is general enough to handle information from any number of end directory services, including LDAP [RFC-1777, RFC-1778], WHOIS++ [RFC-1835], and X.500 [X500].

Directory entries can include far more than just textual information. Some such information (e.g., an image or sound) overlaps with predefined MIME Content-Types. In these cases it can be desirable to include the information in its well-known MIME format. This situation is handled by using a multipart/related Content-Type as defined in [RFC-2112]. The root component of this type is a text/directory body part specifying any in-line information, and for information contained in other Content-Types, the Content-IDs (in URI form) of those parts.

In some applications, it can be useful to include a pointer (e.g, a URI) to some directory information rather than the information itself. This document defines a general mechanism for accomplishing this.

5. The text/directory Content-Type

The text/directory Content-Type is used to hold basic directory information and URIs referencing other information, including other MIME body parts holding supplementary or non-textual directory information, such as an image or sound. It is defined as follows, using the MIME media type registration template from [RFC-2048].

To: ietf-types@uninett.no

Subject: Registration of MIME media type text/directory

\subsection{MIME media type name}

MIME media type name: text 


\subsection{MIME subtype name}

MIME subtype name: directory

\subsection{Required parameters}

Required parameters: charset

The "charset" parameter is as defined in [RFC-2046] for other body parts. It is used to identify the default character set used within the body part.

\subsection{Optional parameters}

Optional parameters: profile

The "profile" parameter is used to convey the type(s) of entity(ies) to which the directory information pertains and the likely set of information associated with the entity(ies). It is intended only as a guide to applications interpreting the information contained within the body part. It SHOULD NOT be used to exclude or require particular pieces of information unless a profile definition specifically calls for this behavior. Unless specifically forbidden by a particular profile definition, a text/directory content type can contain arbitrary attribute/value pairs.

The value of the "profile" parameter is defined as follows. Profile names are case insensitive (i.e., the profile name "vCard" is the same as "VCARD" and "vcard" and "VCArD").

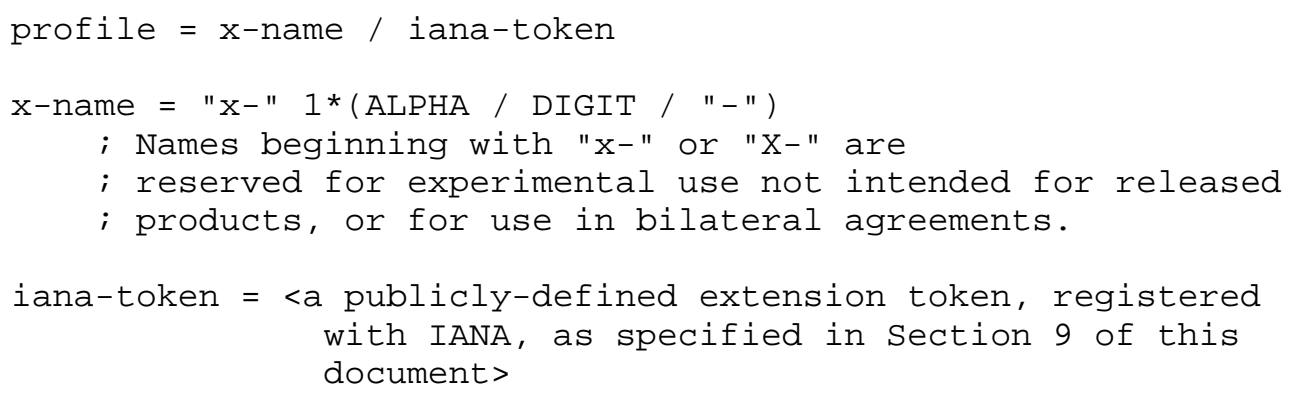




\subsection{Security considerations}

Directory information can be public or it can be protected from unauthorized access by the directory service in which it resides. Once the information leaves its native service, there can be no guarantee that the same care will be taken by all services handling the information. Furthermore, this specification defines no access control mechanism by which information can be protected, or by which access control information can be conveyed. Note that the integrity and privacy of a text/directory body part can be protected by enclosing it within an appropriate MIME-based security mechanism.

\subsection{Interoperability considerations}

In order to make sense of directory information, applications must share a common understanding of the types of information contained within the content-Type (the directory schema). This schema information is not defined in this document, but rather in companion documents (e.g., [MIME-VCARD]) that follow the requirements specified in this document, or in bilateral agreements between communicating parties.

\subsection{Published specification}

The text/directory Content-Type contains directory information, typically pertaining to a single directory entity or group of entities. The content consists of one or more lines in the format given below.

\subsubsection{Line delimiting and folding}

Individual lines within the MIME text/directory Content Type body are delimited by the [RFC-822] line break, which is a CRLF sequence (ASCII decimal 13, followed by ASCII decimal 10). Long logical lines of text can be split into a multiple-physical-line representation using the following folding technique.

A logical line MAY be continued on the next physical line anywhere between two characters by inserting a CRLF immediately followed by a single white space character (space, ASCII decimal 32, or horizontal tab, ASCII decimal 9). At least one character must be present on the folded line. Any sequence of CRLF followed immediately by a single white space character is ignored (removed) when processing the content type. For example the line:

DESCRIPTION:This is a long description that exists on a long line.

Can be represented as: 


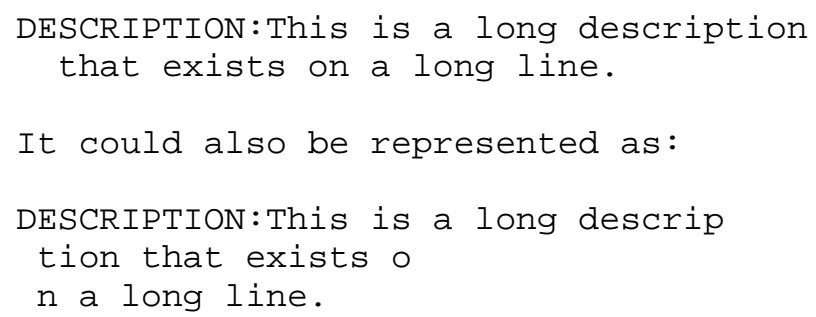

The process of moving from this folded multiple-line representation of a type definition to its single line representation is called unfolding. Unfolding is accomplished by regarding CRLF immediately followed by a white space character (namely HTAB ASCII decimal 9 or SPACE ASCII decimal 32) as equivalent to no characters at all (i.e., the CRLF and single white space character are removed).

\subsubsection{ABNF content-type definition}

The following ABNF uses the notation of RFC 2234, which also defines CRLF, WSP, DQUOTE, VCHAR, ALPHA, and DIGIT. After the unfolding of any folded lines as described above, the syntax for a line of this content type is as follows:

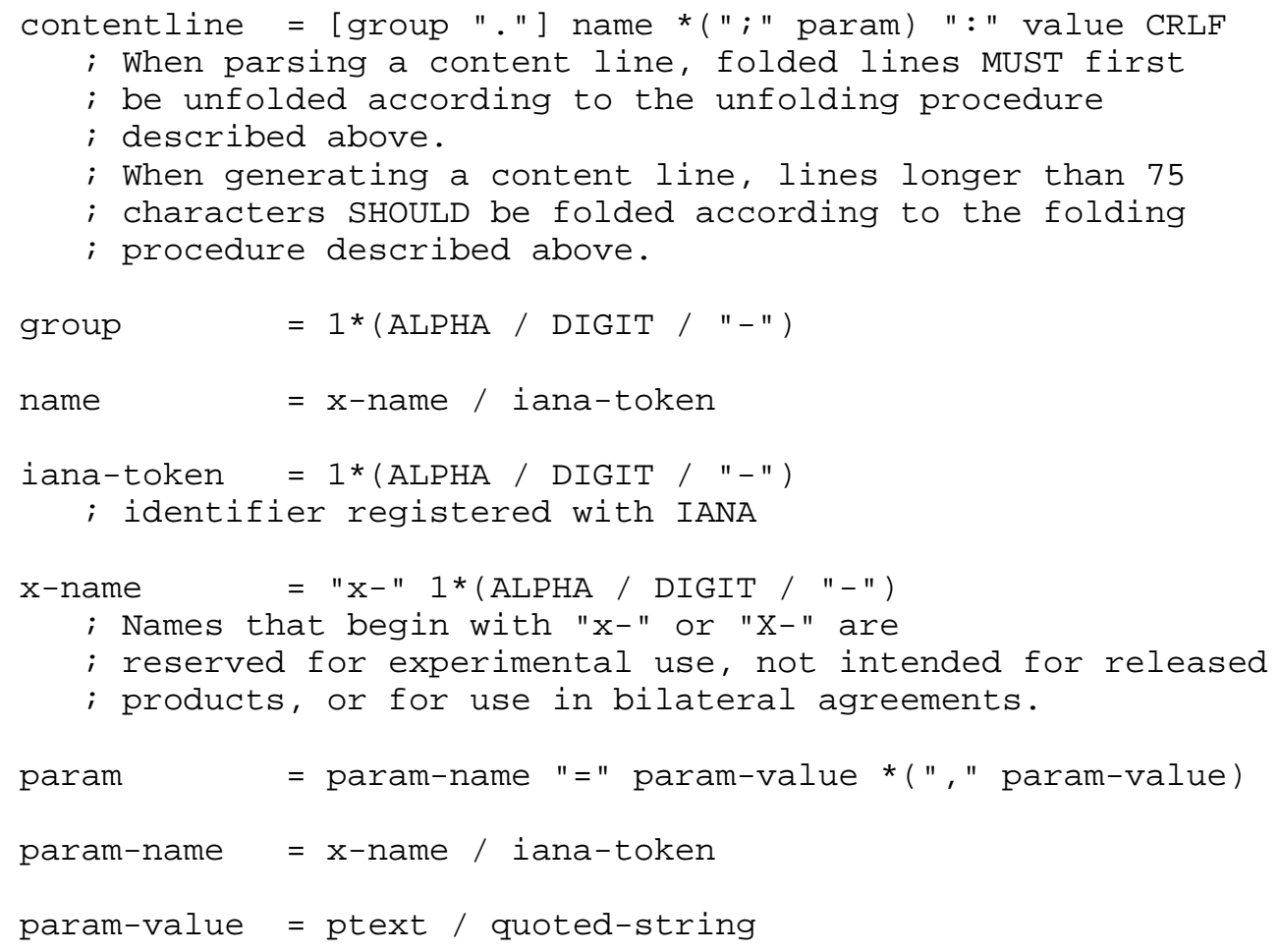




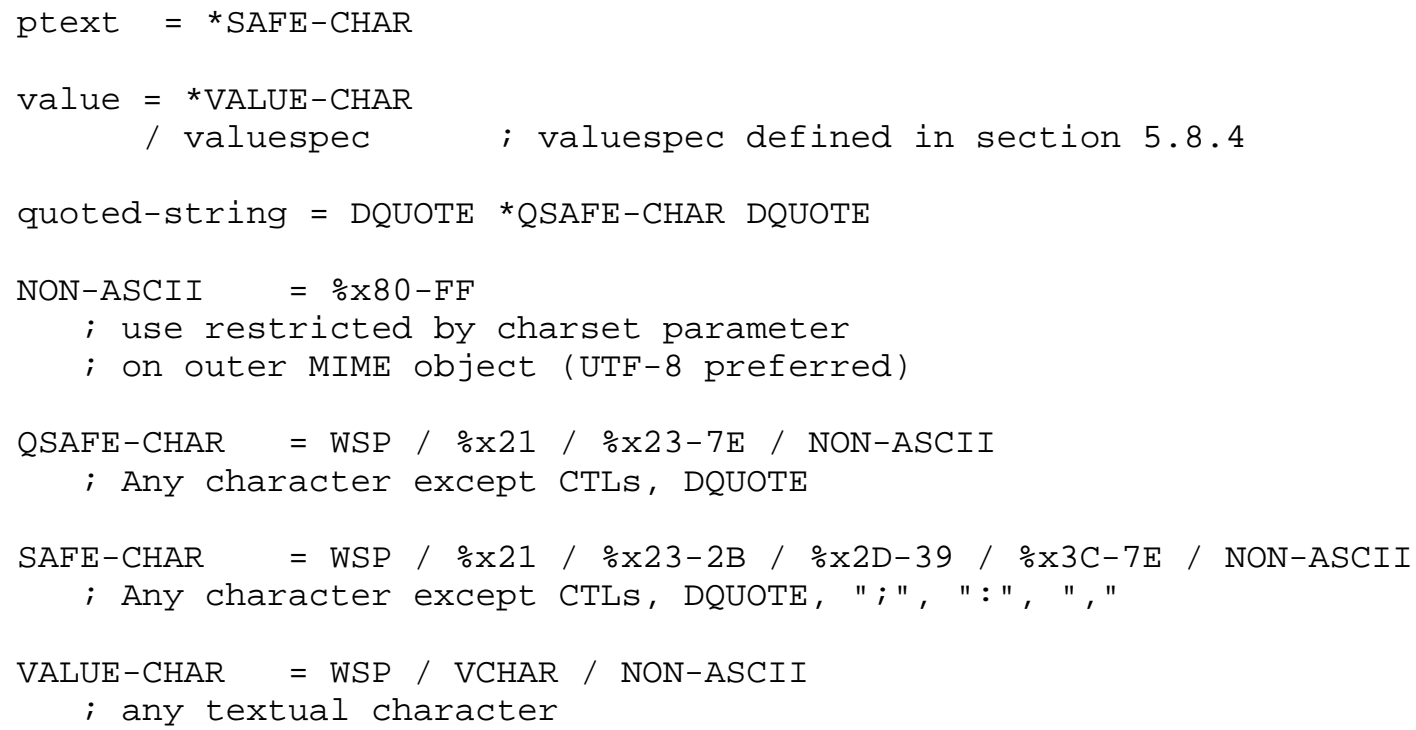

A line that begins with a white space character is a continuation of the previous line, as described above. The white space character and immediately preceeding CRLF should be discarded when reconstructing the original line. Note that this line-folding convention differs from that found in RFC 822, in that the sequence $\langle$ CRLF $\rangle\langle$ WSP $>$ found anywhere in the content indicates a continued line and should be removed.

Various type names and the format of the corresponding values are defined as specified in Section 11. Specifications MAY impose ordering on the type constructs within a body part, though none is required by default. The various $x$-name constructs are used for bilaterally-agreed upon type names, parameter names and parameter values, or for use in experimental settings.

Type names and parameter names are case insensitive (e.g., the type name "fn" is the same as "FN" and "Fn"). Parameter values MAY be case sensitive or case insensitive, depending on their definition.

The group construct is used to group related attributes together. The group name is a syntactic convention used to indicate that all type names prefaced with the same group name SHOULD be grouped together when displayed by an application. It has no other significance. Implementations that do not understand or support grouping MAY simply strip off any text before a "." to the left of the type name and present the types and values as normal. 
Each attribute defined in the text/directory body MAY have multiple values, if allowed in the definition of the profile in which the attribute is used. The general rule for encoding multi-valued items is to simply create a new content line for each value (including the type name). However, it should be noted that some value types support encoding multiple values in a single content line by separating the values with a comma ",". This approach has been taken for several of the content types defined below (date, time, integer, float), for space-saving reasons.

5.8.3. Pre-defined Parameters

The following parameters and value types are defined for general use.

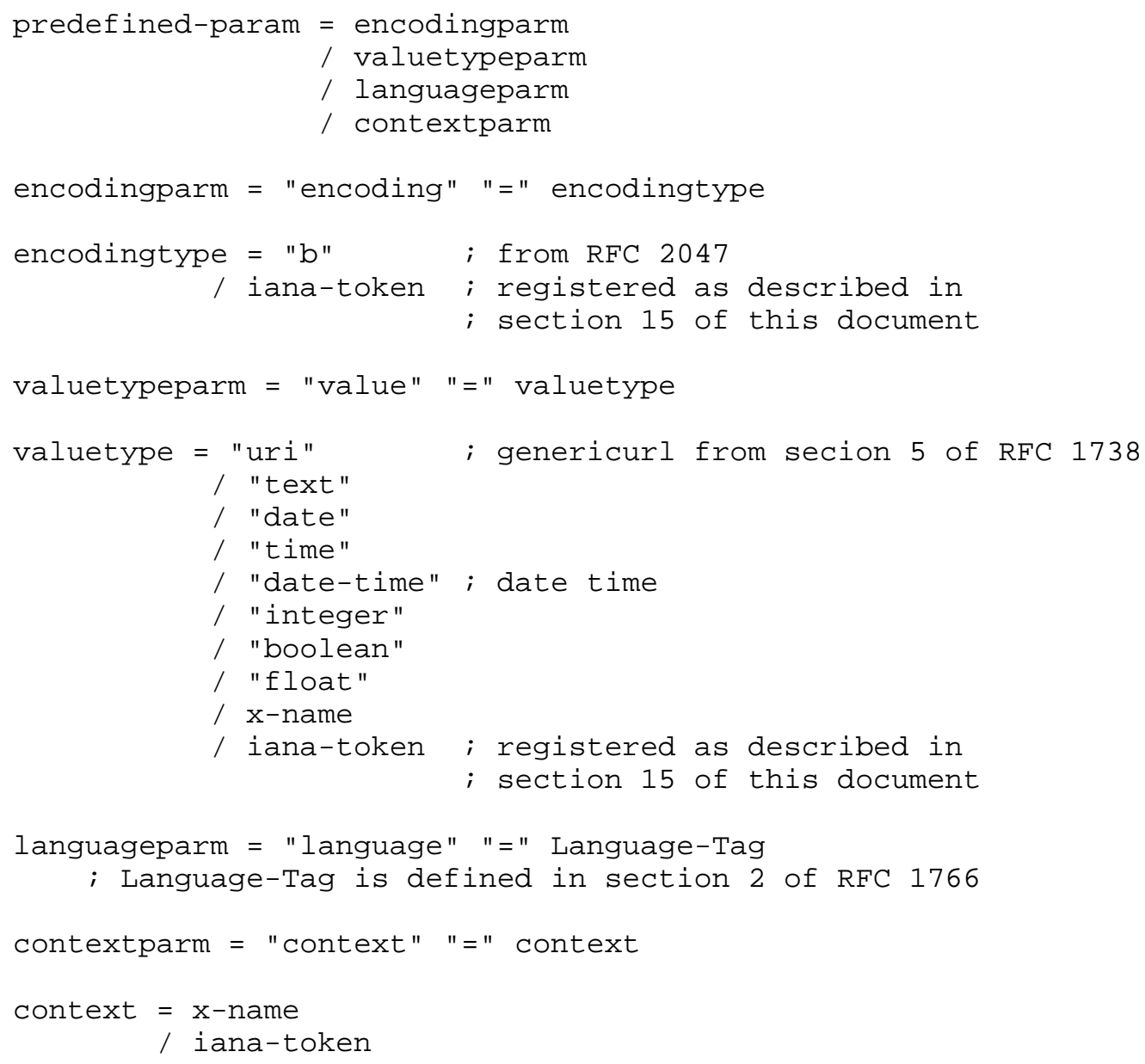


The "language" type parameter is used to identify data in multiple languages. There is no concept of "default" language, except as specified by any "Content-Language" MIME header parameter that is present. The value of the "language" type parameter is a language tag as defined in Section 2 of [RFC-1766].

The "context" type parameter is used to identify a context (e.g., a protocol) used in interpreting the value. This is used, for example, in the "source" type, defined below.

The "encoding" type parameter is used to specify an alternate encoding for a value. If the value contains a CRLF, it must be encoded, since CRLF is used to separate lines in the content-type itself. Currently, only the "b" encoding is supported.

The "b" encoding can also be useful for binary values that are mixed with other text information in the body part (e.g., a certificate). Using a per-value "b" encoding in this case leaves the other information in a more readable form. The encoded base 64 value can be split across multiple physical lines in the content type by using the line folding technique described above.

The Content-Transfer-Encoding header field is used to specify the encoding used for the body part as a whole. The "encoding" type parameter is used to specify an encoding for a particular value (e.g., a certificate). In this case, the content-Transfer-Encoding header might specify "8bit", while the one certificate value might specify an encoding of "b" via an "encoding=b" type parameter.

The Content-Transfer-Encoding and the encodings of individual types given by the "encoding" type parameter are independent of one another. When encoding a text/directory body part for transmission, individual type encodings are performed first, then the entire body part is encoded according to the Content-Transfer-Encoding. When decoding a text/directory body part, the Content-Transfer-Encoding is decoded first, and then any individual types with an "encoding" type parameter are decoded.

The "value" parameter is optional, and is used to identify the value type (data type) and format of the value. The use of these predefined formats is encouraged even if the value parameter is not explicity used. By defining a standard set of value types and their formats, existing parsing and processing code can be leveraged.

Including the value type explicitly as part of each property provides an extra hint to keep parsing simple and support more generalized applications. For example a search engine would not have to know the particular value types for all of the items for which it is 
searching. Because the value type is explicit in the definition, the search engine could look for dates in any item type and provide results that can still be interpreted.

\subsubsection{Pre-defined Value Types}

The format for values corresponding to the predefined valuetype specifications given above are defined.

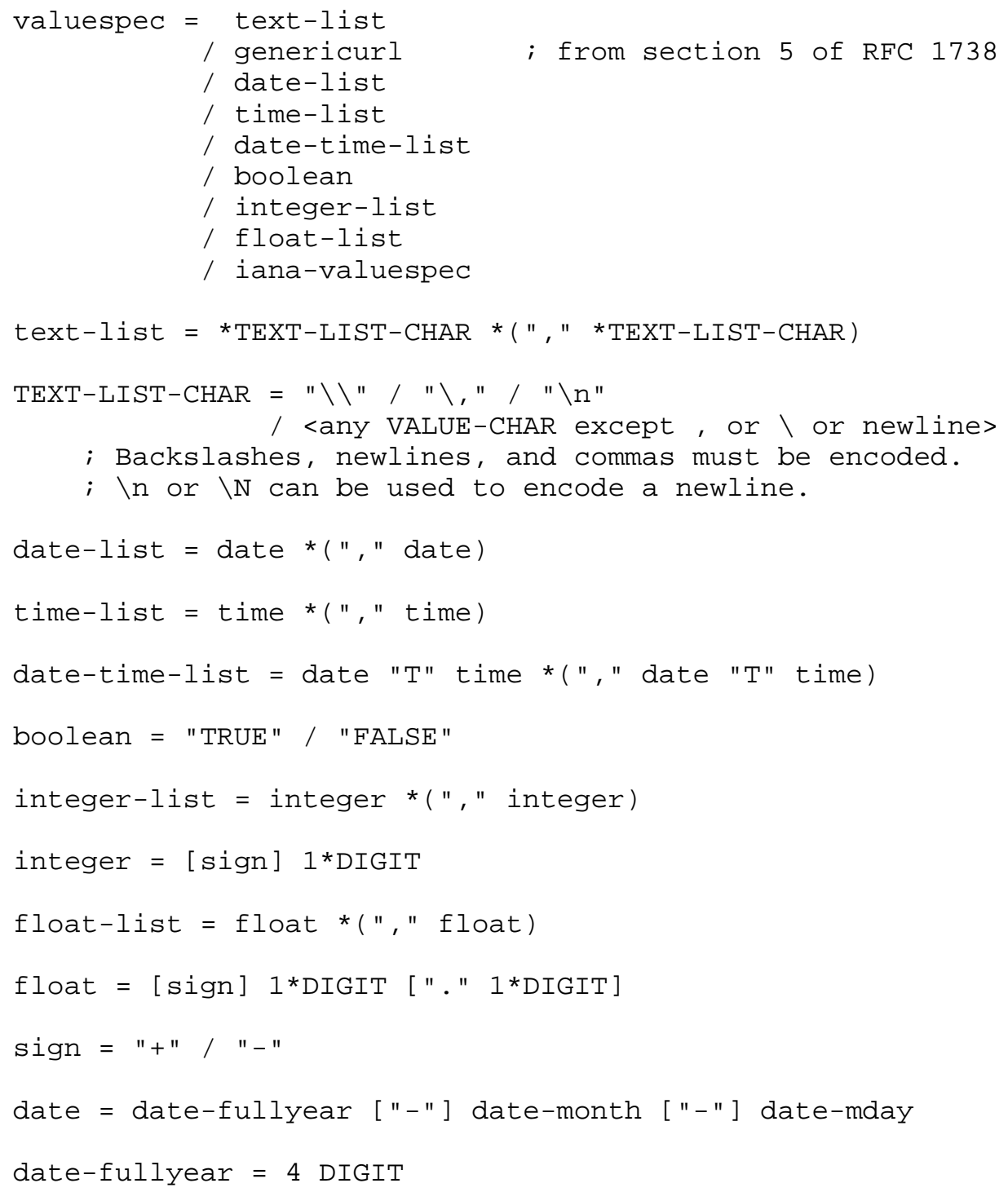




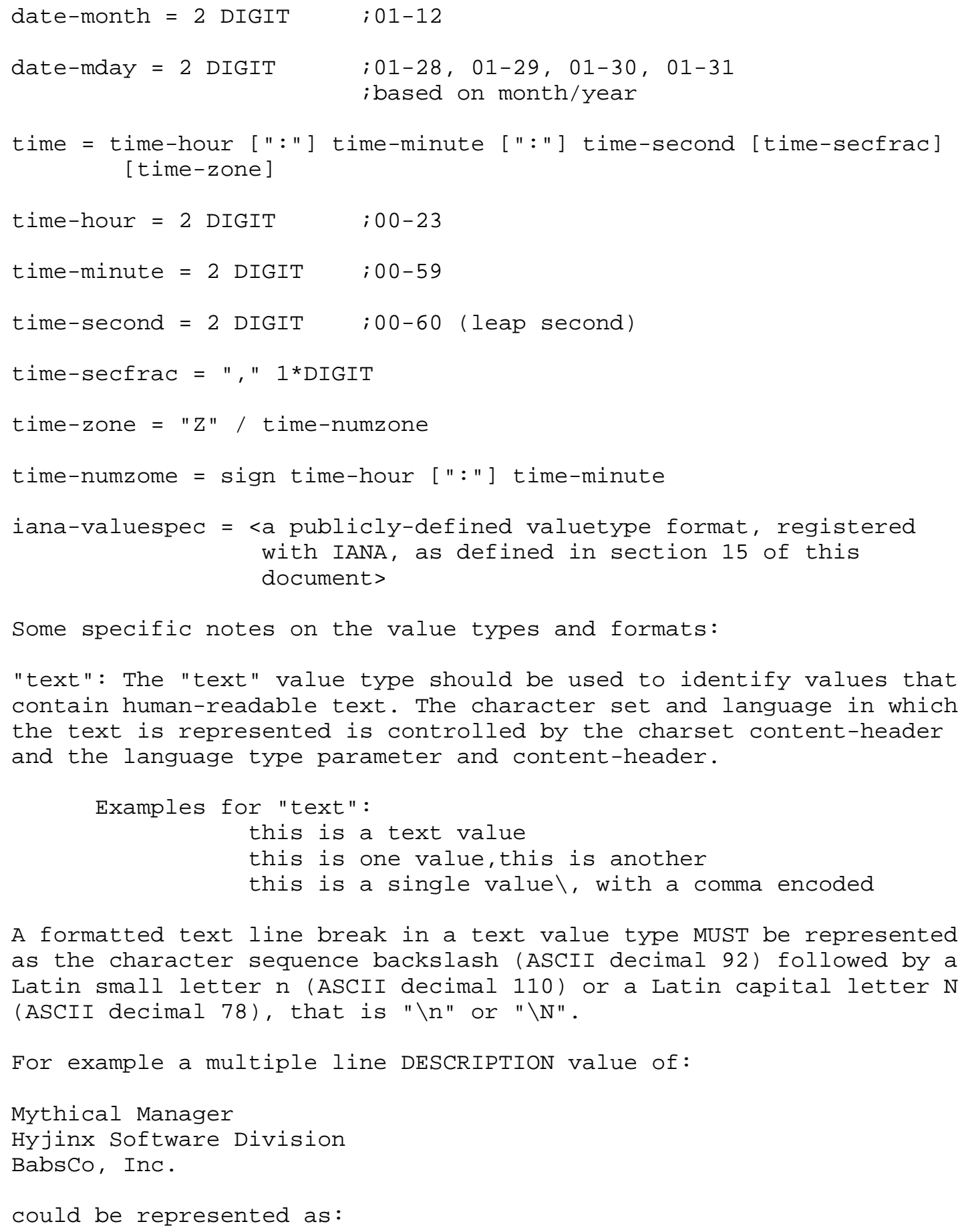




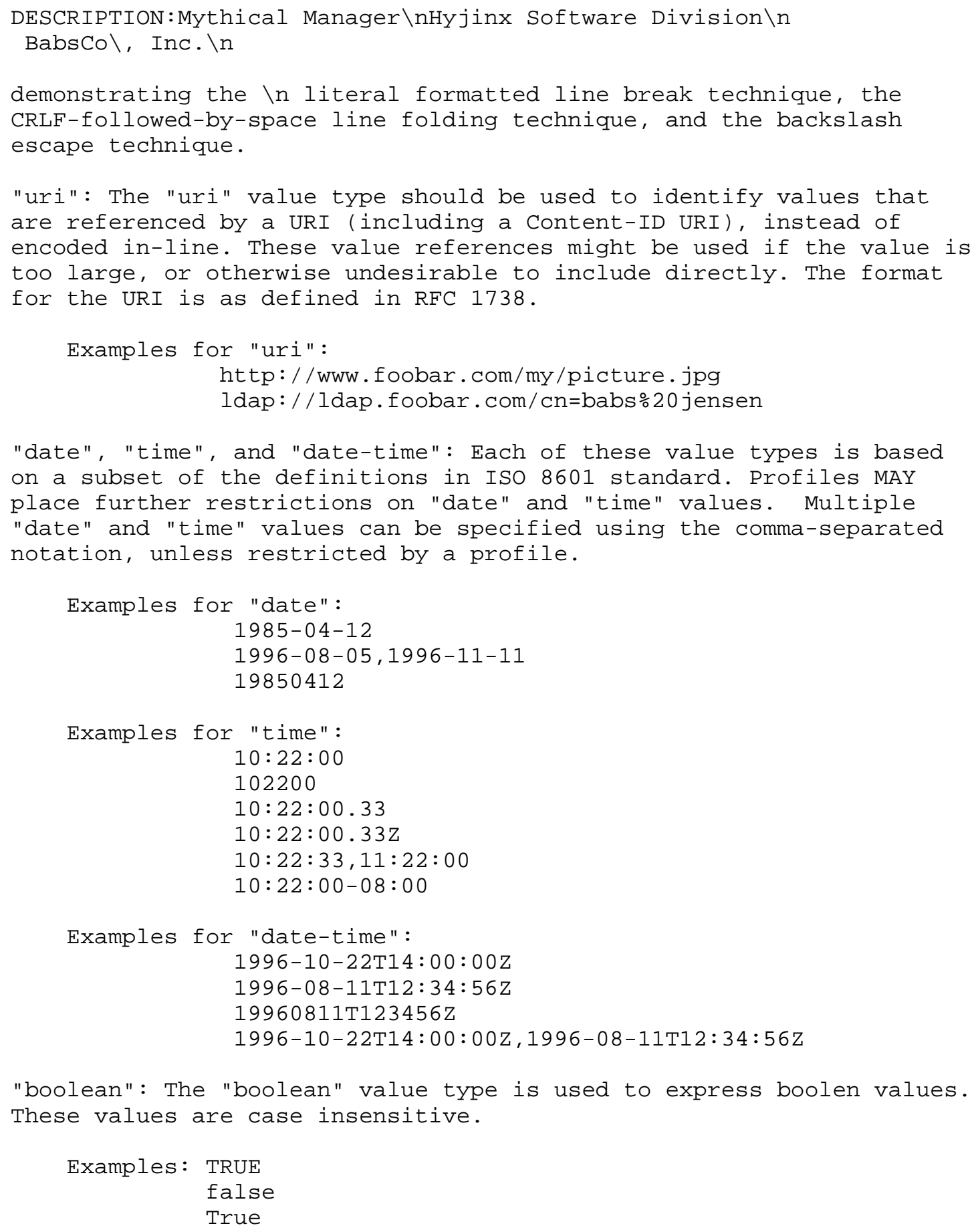


"integer": The "integer" value type is used to express signed integers in decimal format. If sign is not specified, the value is assumed positive "+". Multiple "integer" values can be specified using the comma-separated notation, unless restricted by a profile.

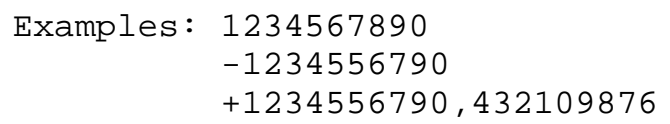

"float": The "float" value type is used to express real numbers. If sign is not specified, the value is assumed positive "+". Multiple "float" values can be specified using the comma-separated notation, unless restricted by a profile.

Examples: 20.30

1000000.0000001

$1.333,3.14$

5.9. Applications which use this media type

Applications which use this media type: Various

5.10. Additional information

Additional information: None

5.11. Person \& email address to contact for further information

Tim Howes

Netscape Communications Corp.

501 East Middlefield Rd.

Mountain View, CA 94041

USA

howes@netscape.com

$+1415937 \quad 3419$

5.12. Intended usage

Intended usage: COMMON 


\subsection{Author/Change controller}

Tim Howes

Netscape Communications Corp.

501 East Middlefield Rd.

Mountain View, CA 94041

USA

howes@netscape.com

$+1415937 \quad 3419$

Mark Smith

Netscape Communications Corp.

501 East Middlefield Rd.

Mountain View, CA 94041

USA

mcs@net scape. com

$+14159373477$

Frank Dawson

Lotus Development Corporation

6544 Battleford Drive

Raleigh, NC 27613-3502

USA

frank_dawson@lotus.com

$+1-919-676-9515$

6. Predefined Types

The following types are generally useful regardless of the profile being carried and are defined below using the text/directory MIME

type registration template defined in Section 11.1 of this document. These types MAY be included in any profile, unless explicitly

forbidden in the profile definition.

\subsection{SOURCE Type Definition}

To: ietf-mime-directeimc.org

Subject: Registration of text/directory MIME type SOURCE

Type name: SOURCE

Type purpose: To identify the source of directory information contained in the content type.

Type encoding: 8bit

Type valuetype: uri 
Type special notes: The SOURCE type is used to provide the means by which applications knowledgable in the given directory service protocol can obtain additional or more up-to-date information from the directory service. It contains a URI as defined in [RFC-1738] and/or other information referencing the directory entity or entities to which the information pertains. When directory information is available from more than one source, the sending entity can pick what it considers to be the best source, or multiple SOURCE types can be included. The interpretation of the value for a SOURCE type can depend on the setting of the CONTEXT type parameter. The value of the CONTEXT type parameter MUST be compatible with the value of the uri prefix.

Type example: SOURCE; CONTEXT=LDAP : 1dap: / / ldap . host / cn=Babs $\% 20$ Jensen, $\div 200=\mathrm{Babsco}, \circ 20 \mathrm{C}=\mathrm{US}$

6.2. NAME Type Definition

To: ietf-mime-directeimc.org

Subject: Registration of text/directory MIME type NAME

Type name: NAME

Type purpose: To identify the displayable name of the directory entity to which information in the content type pertains.

Type encoding: 8bit

Type valuetype: text

Type special notes: The NAME type is used to convey the display name of the entity to which the directory information pertains.

Type example:

NAME:Babs Jensen's Contact Information

\subsection{PROFILE Type Definition}

To: ietf-mime-directeimc.org

Subject: Registration of text/directory MIME type PROFILE

Type name: PROFILE

Type purpose: To identify the type of directory entity to which information in the content type pertains.

Type encoding: 8bit 
Type valuetype: A profile name, registered as described in section 9 of this document or bilaterally agreed upon as described in section 5 .

Type special notes: The PROFILE type is used to convey the type of the entity to which the directory information in the rest of the body part pertains. It should be the same as the "profile" header parameter, if present.

Type example: PROFILE : vCard

6.4. BEGIN Type Definition

To: ietf-mime-directeimc.org

Subject: Registration of text/directory MIME type BEGIN

Type name: BEGIN

Type purpose: To denote the beginning of a syntactic entity within a text/directory content-type.

Type encoding: 8bit

Type valuetype: text, containing a profile name, registered as described in section 9 of this document or bilaterally-agreed upon as described in section 5.

Type special notes: The BEGIN type is used in conjunction with the END type to delimit a profile containing a related set of properties within an text/directory content-type. This construct can be used instead of or in addition to wrapping separate sets of information inside additional MIME headers. It is provided for applications that wish to define content that can contain multiple entities within the same text/directory content-type or to define content that can be identifiable outside of a MIME environment.

Type example: BEGIN : VCARD

6.5. END Type Definition

To: ietf-mime-direct@imc.org

Subject: Registration of text/directory MIME type END

Type name: END 
Type purpose: To denote the end of a syntactic entity within a text/directory content-type.

Type encoding: 8bit

Type valuetype: text, containing a profile name, registered as described in section 9 of this document or bilaterally-agreed upon as described in Section 5.

Type special notes: The END type is used in conjunction with the BEGIN type to delimit a profile containing a related set of properties within an text/directory content-type. This construct can be used instead of or in addition to wrapping separate sets of information inside additional MIME headers. It is provided for applications that wish to define content that can contain multiple entities within the same text/directory content-type or to define content that can be identifiable outside of a MIME environment.

Type example:

END : VCARD

7. Use of the multipart/related Content-Type

The multipart/related Content-Type can be used to hold directory information comprised of both text and non-text information or directory information that already has a natural MIME representation. The root body part within the multipart/related body part is specified as defined in [RFC-2112] by a "start" parameter, or it is the first body part in the absence of such a parameter. The root body part must have a content-Type of "text/directory". This part holds inline information and makes reference to subsequent body parts holding additional text or non-text directory information via their Content-ID URIs as explained in Section 5.

The body parts referred to do not have to be in any particular order, except as noted above for the root body part.

8. Examples

The following examples are for illustrative purposes only and are not part of the definition. 


\subsection{Example 1}

The first example illustrates simple use of the text/directory Content-Type. Note that no "profile" parameter is given, so an application may not know what kind of directory entity the information applies to. Note also the use of both hypothetical official and bilaterally agreed upon types.

From: Whomever@wherever.com

To: Someoned somewhere.com

Subject: whatever

MIME-Version: 1.0

Message-ID: <id1@host. net>

Content-Type: text/directory

Content-ID: <id2@host.com>

cn:Babs Jensen

cn:Barbara J Jensen

sn: Jensen

email: babseumich. edu

phone:+1 313 747-4454

$x-i d: 1234567890$

\subsection{Example 2}

The next example illustrates the use of the Quoted-Printable transfer encoding defined in [RFC 2045] to include non-ASCII character in some of the information returned, and the use of the optional "name" and "source" types. It also illustrates the use of an "encoding" type parameter to encode a certificate value in "b". A "vCard" profile [MIME- VCARD] is used for the example.

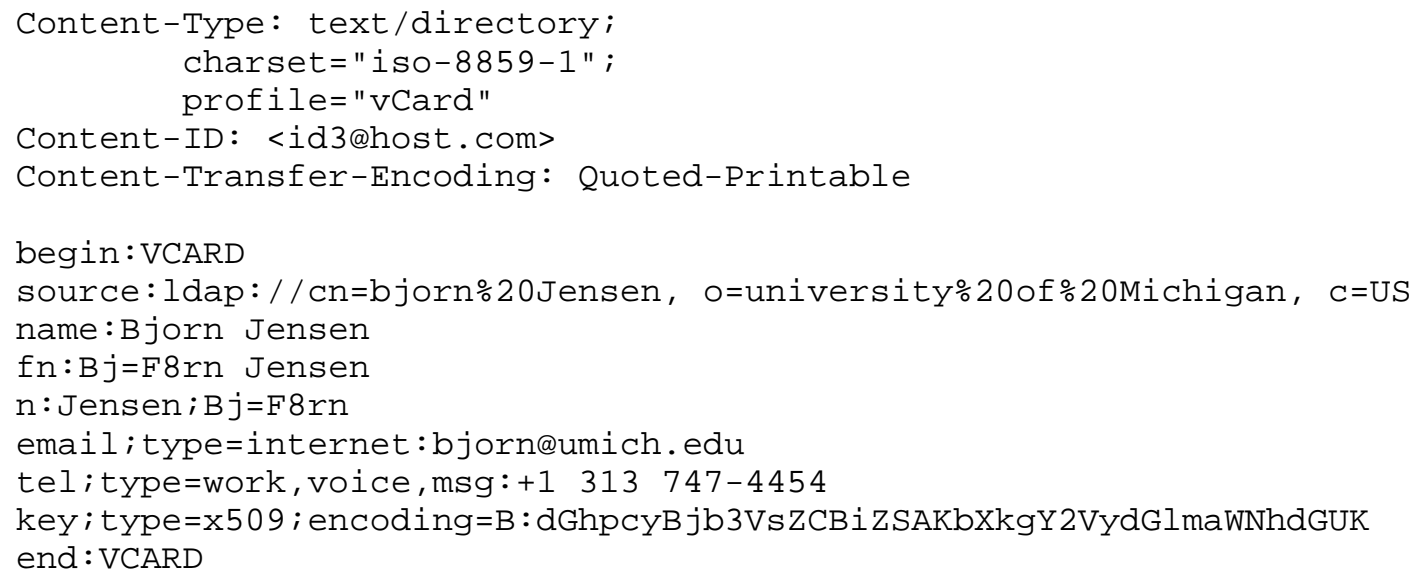




\subsection{Example 3}

The next example illustrates the use of multi-valued type parameters, the "language" type parameter, the "value" type parameter, folding of long lines, the $\backslash n$ encoding for formatted lines, attribute grouping, and the inline "b" encoding. A "vCard" profile [MIME-VCARD] is used for the example.

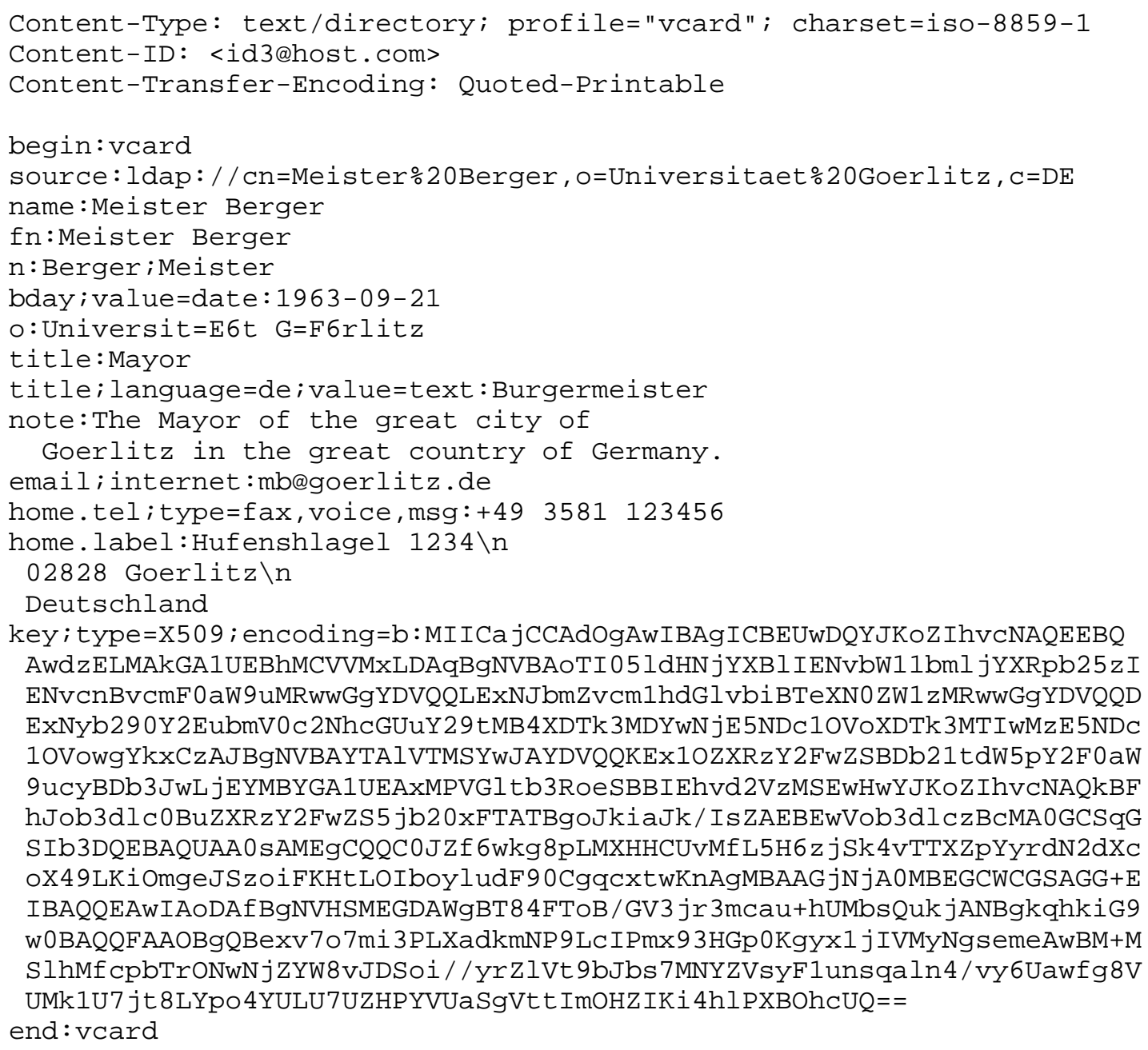




\subsection{Example 4}

The final example illustrates the use of the multipart/related Content-Type to include non-textual directory data via the "uri" encoding to refer to other body parts within the same message, or to external values. Note that no "profile" parameter is given, so an application may not know what kind of directory entity the information applies to. Note also the use of both hypothetical official and bilaterally agreed upon types.

Content-Type: multipart/related; boundary=woof; type="text/directory"; start $="<i d 5 @$ host. com $>"$

Content-ID: <id4@host.com>

-- woof

Content-Type: text/directory; charset="iso-8859-1"

Content-ID: <id5ehost.com>

Content-Transfer-Encoding: Quoted-Printable

source: ldap: / / cn=Bjorn $\% 20$ Jensen, o=University $20 \circ \% 20 \mathrm{Mi}$ chigan, $\mathrm{C}=\mathrm{US}$

$\mathrm{cn}: \mathrm{Bj}=\mathrm{F} 8 \mathrm{rn}$ Jensen

sn: Jensen

email:bjorn@umich.edu

image; value=uri : cid:id6ehost. com

image; value=uri; format=jpeg:ftp://some.host/some/path.jpg

sound; value=uri:cid:id7@host. com

phone:+1 $313 \quad 747-4454$

-- woof

Content-Type: image/jpeg

Content-ID: <id6@host.com>

$<$...image data...>

-- woof

Content-Type: message/external-body;

name="myvoice.au";

site="myhost. com";

access-type=ANON-FTP;

directory="pub/myname" ;

mode=" image"

Content-Type: audio/basic

Content-ID: <id7@host.com>

--woof--

Howes, et. al.

Standards Track

[Page 21] 
9. Registration of new profiles

This section defines procedures by which new profiles are registered with the IANA and made available to the Internet community. Note that non-IANA profiles can be used by bilateral agreement, provided the associated profile names follow the "X-" convention defined above.

The procedures defined here are designed to allow public comment and review of new profiles, while posing only a small impediment to the definition of new profiles.

Registration of a new profile is accomplished by the following steps.

9.1. Define the profile

A profile is defined by completing the following template.

To: ietf-mime-directeimc.org

Subject: Registration of text/directory MIME profile XXX

Profile name:

Profile purpose:

Profile types:

Profile special notes (optional):

Intended usage: (one of COMMON, LIMITED USE or OBSOLETE)

The explanation of what goes in each field in the template follows.

Profile name: The name of the profile as it will appear in the text/directory MIME Content-Type "profile" header parameter, or the predefined "profile" type name.

Profile purpose: The purpose of the profile (e.g., to represent information about people, printers, documents, etc.). Give a short but clear description.

Profile types: The list of types associated with the profile. This list of types is to be expected but not required in the profile, unless otherwise noted in the profile definition. Other types not mentioned in the profile definition MAY also be present. Note that any new types referenced by the profile MUST be defined separately as described in section 10 . 
Profile special notes: Any special notes about the profile, how it is to be used, etc. This section of the template can also be used to define an ordering on the types that appear in the Content-Type, if such an ordering is required.

9.2. Post the profile definition

The profile description must be posted to the new profile discussion list, ietf-mime-directeimc.org

9.3. Allow a comment period

Discussion on the new profile must be allowed to take place on the list for a minimum of two weeks. Consensus must be reached on the profile before proceeding to step 4 .

9.4. Submit the profile for approval

Once the two-week comment period has elapsed, and the proposer is convinced consensus has been reached on the profile, the registration application should be submitted to the Profile Reviewer for approval. The Profile Reviewer is appointed by the Application Area Directors and can either accept or reject the profile registration. An accepted registration is passed on by the Profile Reviewer to the IANA for inclusion in the official IANA profile registry. The registration may be rejected for any of the following reasons. 1) Insufficient comment period; 2) Consensus not reached; 3) Technical deficiencies raised on the list or elsewhere have not been addressed. The Profile Reviewer's decision to reject a profile can be appealed by the proposer to the IESG, or the objections raised can be addressed by the proposer and the profile resubmitted.

10. Profile Change Control

Existing profiles can be changed using the same process by which they were registered.

Define the change

Post the change

Allow a comment period

Submit the changed profile for approval 
Note that the original author or any other interested party can propose a change to an existing profile, but that such changes should only be proposed when there are serious omissions or errors in the published specification. The Profile Reviewer can object to a change if it is not backwards compatible, but is not required to do so.

Profile definitions can never be deleted from the IANA registry, but profiles which are no longer believed to be useful can be declared OBSOLETE by a change to their "intended use" field.

11. Registration of new types

This section defines procedures by which new types are registered with the IANA. Note that non-IANA types can be used by bilateral agreement, provided the associated types names follow the "X-" convention defined above.

The procedures defined here are designed to allow public comment and review of new types, while posing only a small impediment to the definition of new types.

Registration of a new type is accomplished by the following steps.

11.1. Define the type

A type is defined by completing the following template.

To: ietf-mime-directeimc.org

Subject: Registration of text/directory MIME type XXX

Type name:

Type purpose:

Type encoding:

Type valuetype:

Type special notes (optional):

Intended usage: (one of COMMON, LIMITED USE or OBSOLETE)

The meaning of each field in the template is as follows.

Type name: The name of the type, as it will appear in the body of an text/directory MIME Content-Type "type: value" line to the left of the colon ":". 
Type purpose: The purpose of the type (e.g., to represent a name, postal address, IP address, etc.). Give a short but clear description.

Type encoding: The default encoding a value of the type must have in the body of a text/directory MIME Content-Type.

Type valuetype: The format a value of the type must have in the body of a text/directory MIME Content-Type. This description must be precise and must not violate the general encoding rules defined in section 5 of this document.

Type special notes: Any special notes about the type, how it is to be used, etc.

11.2. Post the type definition

The type description must be posted to the new type discussion list, ietf-mime-direct@imc.org

11.3. Allow a comment period

Discussion on the new type must be allowed to take place on the list for a minimum of two weeks. Consensus must be reached on the type before proceeding to step 4 .

11.4. Submit the type for approval

Once the two-week comment period has elapsed, and the proposer is convinced consensus has been reached on the type, the registration application should be submitted to the Profile Reviewer for approval. The Profile Reviewer is appointed by the Application Area Directors and can either accept or reject the type registration. An accepted registration is passed on by the Profile Reviewer to the IANA for inclusion in the official IANA profile registry. The registration can be rejected for any of the following reasons. 1) Insufficient comment period; 2) Consensus not reached; 3) Technical deficiencies raised on the list or elsewhere have not been addressed. The Profile Reviewer's decision to reject a type can be appealed by the proposer to the IESG, or the objections raised can be addressed by the proposer and the type resubmitted. 


\section{Type Change Control}

Existing types can be changed using the same process by which they were registered.

Define the change

Post the change

Allow a comment period

Submit the type for approval

Note that the original author or any other interested party can propose a change to an existing type, but that such changes should only be proposed when there are serious omissions or errors in the published specification. The Profile Reviewer can object to a change if it is not backwards compatible, but is not required to do so.

Type definitions can never be deleted from the IANA registry, but types which are nolonger believed to be useful can be declared OBSOLETE by a change to their "intended use" field.

13. Registration of new parameters

This section defines procedures by which new parameters are registered with the IANA and made available to the Internet community. Note that non-IANA parameters can be used by bilateral agreement, provided the associated parameters names follow the "X-" convention defined above.

The procedures defined here are designed to allow public comment and review of new parameters, while posing only a small impediment to the definition of new parameters.

Registration of a new parameter is accomplished by the following steps.

\subsection{Define the parameter}

A parameter is defined by completing the following template.

To: ietf-mime-direct@imc.org

Subject: Registration of text/directory MIME type parameter XXX

Parameter name:

Parameter purpose: 


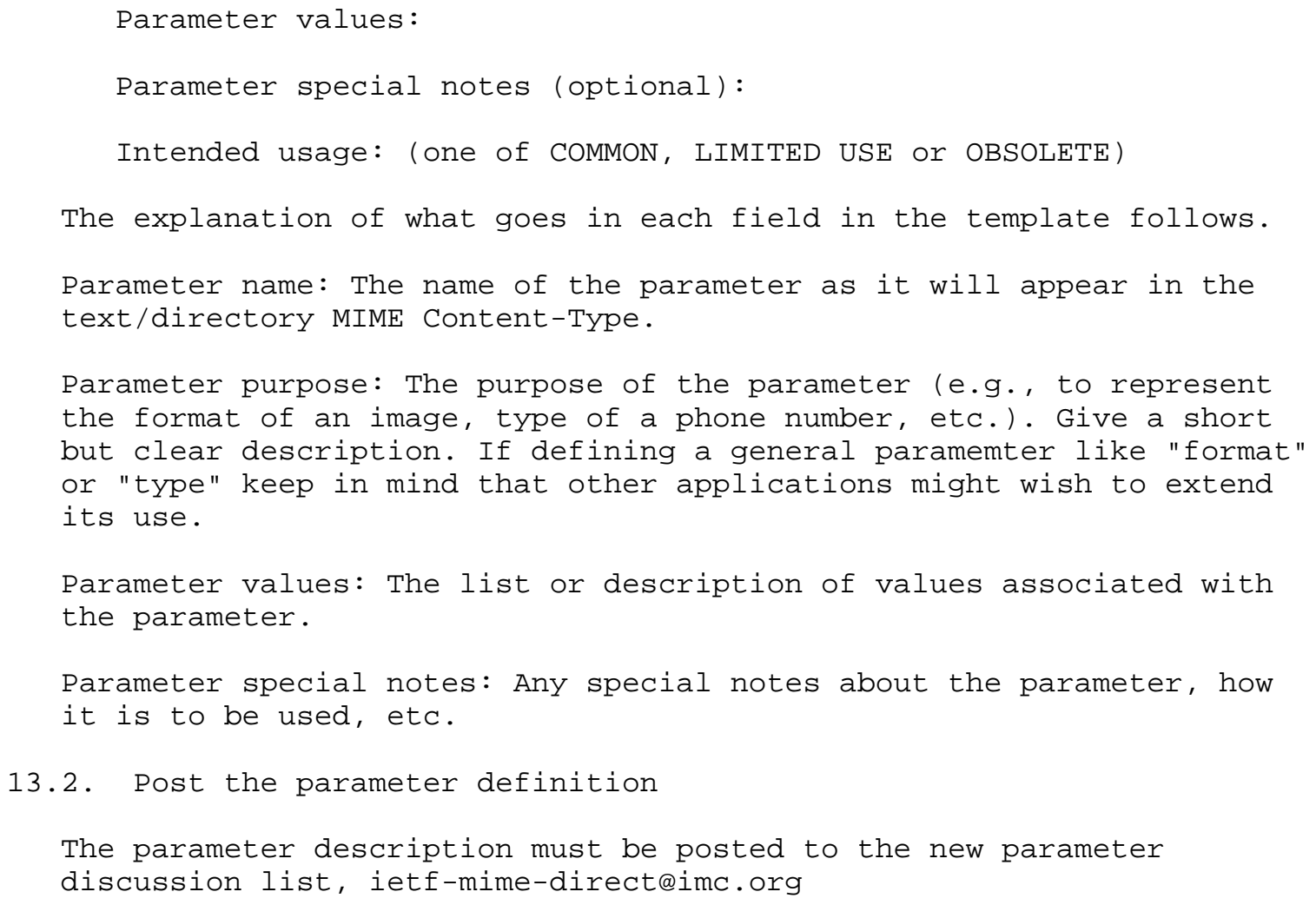

13.4. Submit the parameter for approval

Once the two-week comment period has elapsed, and the proposer is convinced consensus has been reached on the parameter, the registration application should be submitted to the Profile Reviewer for approval. The Profile Reviewer is appointed by the Application Area Directors and can either accept or reject the parameter registration. An accepted registration is passed on by the Profile Reviewer to the IANA for inclusion in the official IANA parameter registry. The registration can be rejected for any of the following reasons. 1) Insufficient comment period; 2) Consensus not reached; 3) Technical deficiencies raised on the list or elsewhere have not been addressed. The Profile Reviewer's decision to reject a profile can be appealed by the proposer to the IESG, or the objections raised can be 
addressed by the proposer and the parameter registration resubmitted.

14. Parameter Change Control

Existing parameters can be changed using the same process by which they were registered.

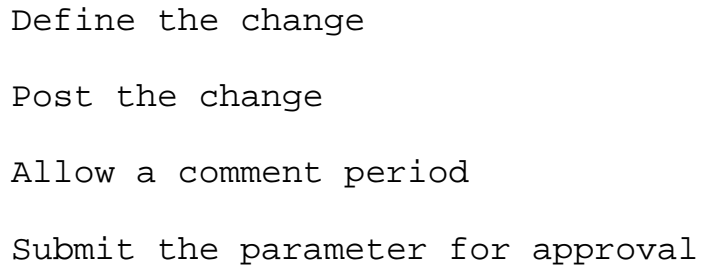

15. Registration of new value types

This section defines procedures by which new value types are registered with the IANA and made available to the Internet community. Note that non-IANA value types can be used by bilateral agreement, provided the associated value types names follow the "X-" convention defined above.

The procedures defined here are designed to allow public comment and review of new value types, while posing only a small impediment to the definition of new value types.

Registration of a new value types is accomplished by the following steps.

15.1. Define the value type

A value type is defined by completing the following template.

To: ietf-mime-directeimc.org

Subject: Registration of text/directory MIME value type XXX 


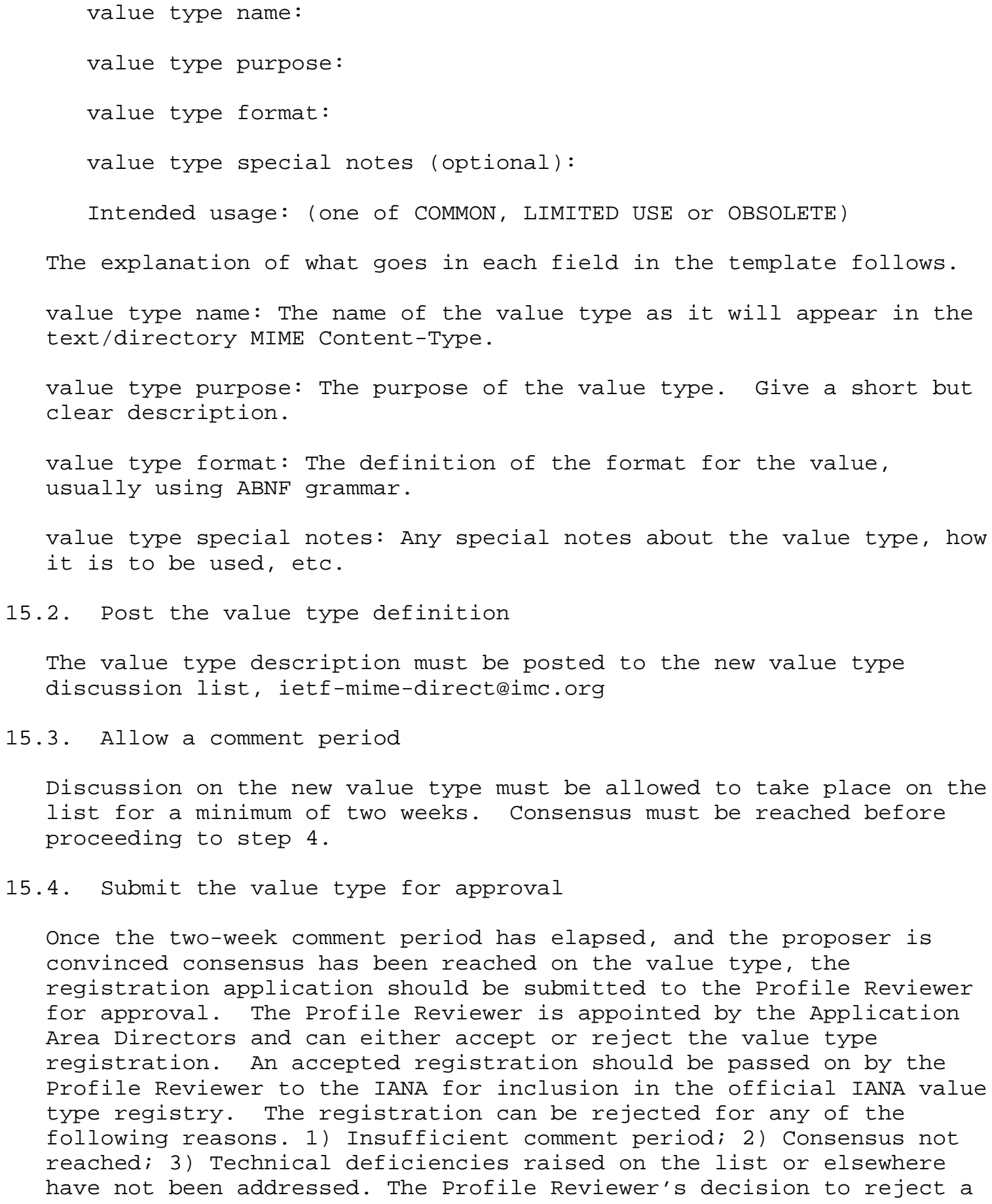


profile can be appealed by the proposer to the IESG, or the objections raised can be addressed by the proposer and the value type registration resubmitted.

\section{Security Considerations}

Internet mail is subject to many well known security attacks, including monitoring, replay, and forgery. Care should be taken by any directory service in allowing information to leave the scope of the service itself, where any access controls can no longer be guaranteed. Applications should also take care to display directory data in a "safe" environment (e.g., PostScript-valued types).

\section{Acknowledgements}

The registration procedures defined here were shamelessly lifted from the MIME registration RFC.

The many valuable comments contributed by members of the IETF ASID working group are gratefully acknowledged, as are the contributions of the Versit Consortium. Chris Newman was especially helpful in navigating the intricacies of $\mathrm{ABNF}$ lore.

18. References

[RFC-1777] Yeong, W., Howes, T., and S. Kille, "Lightweight Directory Access Protocol", RFC 1777, March 1995.

[RFC-1778] Howes, T., Kille, S., Yeong, W., and C. Robbins, "The String Representation of Standard Attribute Syntaxes", RFC 1778, March 1995.

[RFC-822] Crocker, D., "Standard for the Format of ARPA Internet Text Messages", STD 11, RFC 822, August 1982.

[RFC-2045] Borenstein, N., and N. Freed, "Multipurpose Internet Mail Extensions (MIME) Part One: Format of Internet Message Bodies", RFC 2045, November 1996.

[RFC-2046] Moore, K., "Multipurpose Internet Mail Extensions (MIME) Part Two: Media Types", RFC 2046, November 1996.

[RFC-2048] Freed, N., Klensin, J., and J. Postel, "Multipurpose Internet Mail Extensions (MIME) Part Four: Registration Procedures", RFC 2048, November 1996.

[RFC-1766] Alvestrand, H., "Tags for the Identification of Languages", RFC 1766, March 1995. 


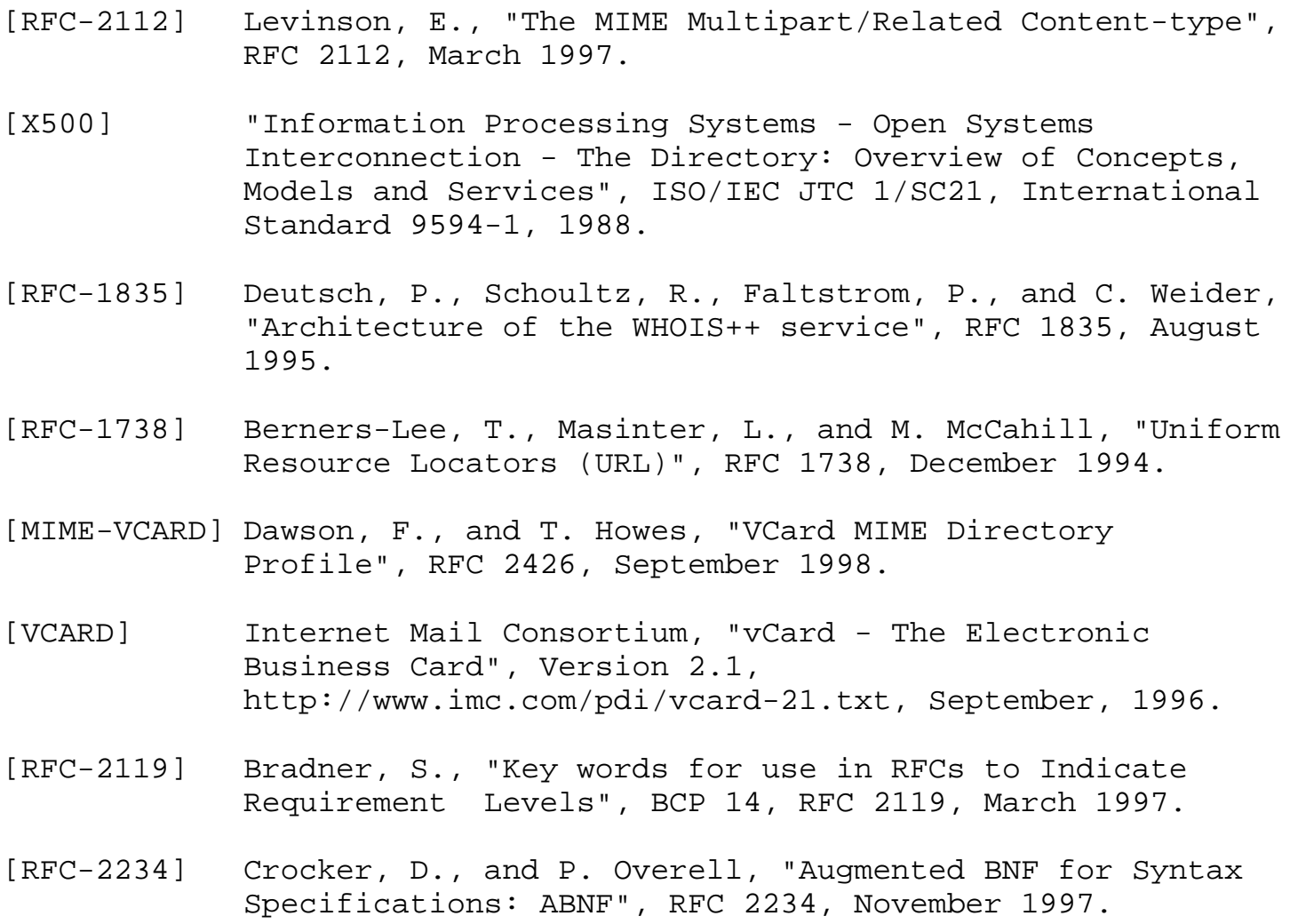


19. Authors' Addresses

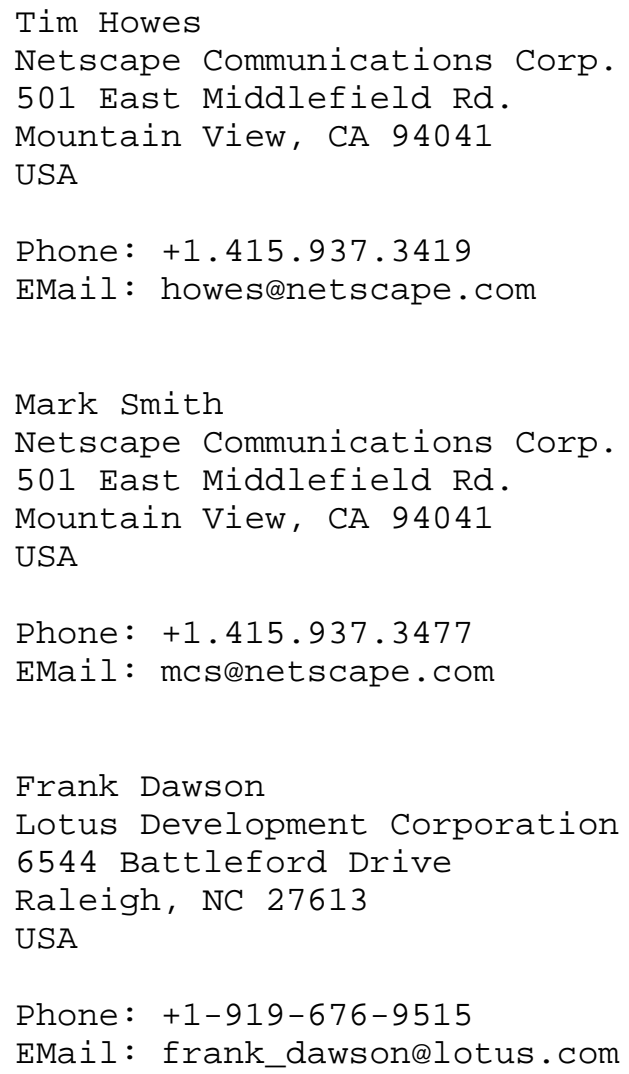


20. Full Copyright statement

Copyright (C) The Internet Society (1998). All Rights Reserved.

This document and translations of it may be copied and furnished to others, and derivative works that comment on or otherwise explain it or assist in its implementation may be prepared, copied, published and distributed, in whole or in part, without restriction of any kind, provided that the above copyright notice and this paragraph are included on all such copies and derivative works. However, this document itself may not be modified in any way, such as by removing the copyright notice or references to the Internet society or other Internet organizations, except as needed for the purpose of developing Internet standards in which case the procedures for copyrights defined in the Internet Standards process must be followed, or as required to translate it into languages other than English.

The limited permissions granted above are perpetual and will not be revoked by the Internet society or its successors or assigns.

This document and the information contained herein is provided on an "AS IS" basis and THE INTERNET SOCIETY AND THE INTERNET ENGINEERING TASK FORCE DISCLAIMS ALL WARRANTIES, EXPRESS OR IMPLIED, INCLUDING BUT NOT LIMITED TO ANY WARRANTY THAT THE USE OF THE INFORMATION HEREIN WILL NOT INFRINGE ANY RIGHTS OR ANY IMPLIED WARRANTIES OF MERCHANTABILITY OR FITNESS FOR A PARTICULAR PURPOSE. 